\title{
Mechanical Design and Kinematics Analysis of a Hydraulically Actuated Manipulator
}

\author{
Xuewen Rong ${ }^{1}$, Rui Song ${ }^{*}$, , Hui Chai ${ }^{1}$ and Xiaolin $\mathrm{Ma}^{2}$ \\ ${ }^{1}$ School of Control Science and Engineering, Shandong University, Jinan 250061, China \\ ${ }^{2}$ School of Mechanical and Electrical Engineering, Shandong Jianzhu University, Jinan 250101, China
}

\begin{abstract}
This paper gives a mechanism design of a six DOF hydraulically actuated manipulator firstly. Then its DH frames and link parameters are given. Next, its forward kinematic equations are derived according to homogeneous transformation method. Fourthly, the analytical solutions of its inverse kinematics are solved by given the position and posture of the end-effector simultaneously. The posture of the end-effector is given with three $z-y-z$ Euler angles for they have obvious geometry meanings and are easy to be measured. In addition, the correctness of the inverse kinematic equations is verified in Simulink by comparing many sets of randomly produced joint variables in workspace and their corresponding inverse solutions.
\end{abstract}

Keywords: DH frame, DOF, Euler angle, homogeneous transformation, hydraulic, manipulator.

\section{INTRODUCTION}

The traditional electric powered industrial manipulators have been used in all kinds of industrial productions. They are already very mature in technology and with high precision. But they are not suitable for several tasks in severe environments for their electric drive mode and low powermass ratio, such as underwater, construction, electric power lines maintenance, etc. In order to make up for the inadequacy of electric powered manipulators, some hydraulically actuated multi-joints manipulators have been developed in several countries.

Uninterrupted power supply has become indispensable during the maintenance task of active electric power lines as a result of today's highly information-oriented society and increasing demand of electric utilities. The maintenance task has the risk of electric shock and the danger of falling from high place. Therefore, it is necessary to realize an autonomous robot system using electro-hydraulic manipulator because hydraulic manipulators have the advantage of electric insulation and high power-mass ratio [1, 2]. Dunnigan et al. [3-5] introduce several hydraulic manipulators working for underwater, forest and construction tasks respectively. Taylor et al. [6,7] introduce two kinds of hydraulic manipulators for nuclear industries. Wang et al. [8] introduces a hydraulic manipulator developed in China but there is no application case given. Zhao Y. L. gives two application cases of hydraulic manipulators using in electric power line maintenance $[9,10]$.

This paper gives the mechanical structure of a six DOF hydraulic manipulator in section 2, which is named HydM and developed by Shandong University, China. In section 3,

*Address correspondence to this author at the School of Control Science and Engineering, Shandong University, Jinan 250061, China; Tel: 0086531-88396813; Fax: 0086-531-88396813; E-mail: rsong@sdu.edu.cn the forward kinematic equations are given firstly. Then the analytical solutions of its inverse kinematics are solved by given the position and posture of the end-effector simultaneously.

\section{MECHANISM DESIGN OF HydM}

The mechanical structure sketch of HydM is shown in Fig. (1). HydM has six rotary degrees of freedom (DOF) which are all driven by hydraulic actuators. The first, the fourth and the fifth joints are driven by helical rotary actuators. The second and the third joints are driven by hydraulic linear cylinders. The sixth joint is driven by a miniature hydraulic motor through a worm reducer, so it can turn continuously. In fact, the end-effector is a gripper and its opening and closing movement is driven by a miniature linear cylinder. The movement limits of each joint are listed in Table 1. The movements of all the six joints and the gripper are controlled by servo valves which are mounted on a

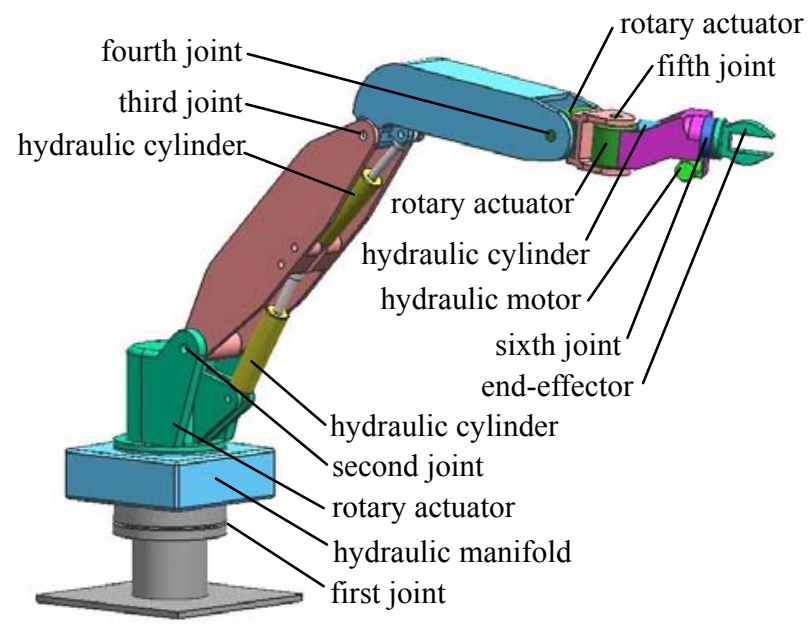

Fig. (1). The mechanical structure sketch of HydM. 
hydraulic manifold. HydM does not integrate with a hydraulic power unit, so it must be supplied hydraulic power from an external hydraulic power unit through high pressure hose.

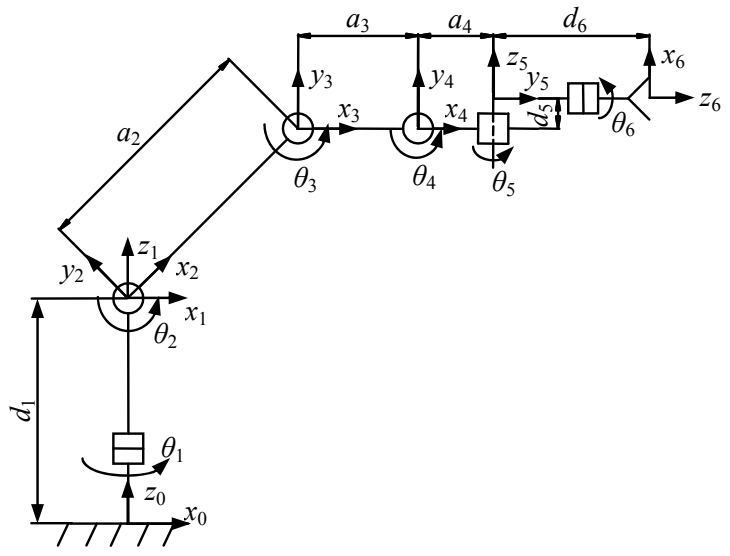

Fig. (2). The DH frames of HydM.

\section{KINEMATICS ANALYSIS OF HydM}

\subsection{The DH Frames and Link Parameters of HydM}

According to the DH rules [11], the global frame $\left\{O_{0}\right\}$ and local frames $\left\{O_{i}\right\}$ fixed to each link can be build as shown in Fig. (2).

Then the link parameters in DH frames of each link can be gained as listed in Table 1. The open intervals in last column show the motion range of each joint.

\subsection{Forward Kinematics of HydM}

The homogeneous transformation method based DH rules is the most commonly used method to solve the forward kinematics of manipulators or other complex serial mechanisms. According to Fig. (1) and Table 1, it is easy to get the homogeneous transformation matrix between two adjacent links. The HydM manipulator has six joints and seven links including the mounting base, so there have six homogeneous transformation matrices. The successive product of these matrices describes the position and posture of the end-effector in global frame. And it is also the forward kinematic equation of the manipulator shown as Eq. (1).

${ }^{0} \mathbf{T}_{6}={ }^{0} \mathbf{T}_{1} \cdot{ }^{1} \mathbf{T}_{2} \cdot{ }^{2} \mathbf{T}_{3} \cdot{ }^{3} \mathbf{T}_{4} \cdot{ }^{4} \mathbf{T}_{5} \cdot{ }^{5} \mathbf{T}_{6}=\left[\begin{array}{cccc}n_{x} & o_{x} & a_{x} & p_{x} \\ n_{y} & o_{y} & a_{y} & p_{y} \\ n_{z} & o_{z} & a_{z} & p_{z} \\ 0 & 0 & 0 & 1\end{array}\right]$

$n_{x}=c_{1} c_{234} c_{5} c_{6}-s_{1} S_{5} c_{6}+c_{1} S_{234} s_{6}, n_{y}=s_{1} c_{234} c_{5} c_{6}+c_{1} S_{5} c_{6}+s_{1} S_{234} S_{6}$,

$n_{z}=s_{234} c_{5} c_{6}-c_{234} S_{6}, o_{x}=-c_{1} c_{234} c_{5} S_{6}+S_{1} S_{5} S_{6}+c_{1} S_{234} c_{6}$,

$o_{y}=-S_{1} c_{234} c_{5} S_{6}-c_{1} S_{5} s_{6}+S_{1} S_{234} c_{6}, o_{z}=-S_{234} c_{5} s_{6}-c_{234} c_{6}$,

$a_{x}=-c_{1} c_{234} s_{5}-s_{1} c_{5}, a_{y}=-S_{1} c_{234} s_{5}+c_{1} c_{5}$,

$a_{z}=-s_{234} s_{5}, p_{x}=c_{1}\left(a_{2} c_{2}+a_{3} c_{23}+a_{4} c_{234}-d_{5} s_{234}\right)-d_{6}\left(c_{1} c_{234} s_{5}+s_{1} c_{5}\right)$, $p_{y}=s_{1}\left(a_{2} c_{2}+a_{3} c_{23}+a_{4} c_{234}-d_{5} s_{234}\right)-d_{6}\left(s_{1} c_{234} s_{5}-c_{1} c_{5}\right)$,

$p_{z}=a_{2} s_{2}+d_{1}+a_{3} s_{23}+a_{4} s_{234}+d_{5} c_{234}-d_{6} s_{234} s_{5}$,

where,

$s_{i}=\sin \theta_{i}, \quad c_{i}=\cos \theta_{i}, \quad s_{i j}=\sin \left(\theta_{i}+\theta_{j}\right), \quad c_{i j}=\cos \left(\theta_{i}+\theta_{j}\right)$,

$s_{i j k}=\sin \left(\theta_{i}+\theta_{j}+\theta_{k}\right), \quad c_{i j k}=\cos \left(\theta_{i}+\theta_{j}+\theta_{k}\right)$.

Table 1. Link parameters of HydM.

\begin{tabular}{|c|c|c|c|c|c|c|}
\hline $\begin{array}{c}\text { Link No. } \\
\boldsymbol{i}\end{array}$ & $\begin{array}{c}\boldsymbol{a}_{\boldsymbol{i}-1} \\
(\mathbf{m m})\end{array}$ & $\boldsymbol{\alpha}_{\boldsymbol{i}-1}$ & $\begin{array}{c}\boldsymbol{d}_{\boldsymbol{i}} \\
(\mathbf{m m})\end{array}$ & $\boldsymbol{\theta}_{\boldsymbol{i}}$ & $\begin{array}{c}\text { Joint } \\
\text { Variable }\end{array}$ & Limits \\
\hline \hline 1 & 0 & 0 & $d_{1}$ & $\theta_{1}$ & $\theta_{1}$ & $\left(-90^{\circ}, 90^{\circ}\right)$ \\
\hline 2 & 0 & $90^{\circ}$ & 0 & $\theta_{2}$ & $\theta_{2}$ & $\left(0^{\circ}, 120^{\circ}\right)$ \\
\hline 3 & $a_{2}$ & 0 & 0 & $\theta_{3}$ & $\theta_{3}$ & $\left(-125^{\circ},-5^{\circ}\right)$ \\
\hline 4 & $a_{3}$ & 0 & 0 & $\theta_{4}$ & $\theta_{4}$ & $\left(-50^{\circ}, 70^{\circ}\right)$ \\
\hline 5 & $a_{4}$ & $-90^{\circ}$ & $d_{5}$ & $\theta_{5}$ & $\theta_{5}$ & $\left(-150^{\circ},-30^{\circ}\right)$ \\
\hline 6 & 0 & $-90^{\circ}$ & $d_{6}$ & $\theta_{6}$ & $\theta_{6}$ & $\left(-180^{\circ}, 180^{\circ}\right)$ \\
\hline
\end{tabular}

\subsection{Inverse Kinematics of HydM}

For manipulators, inverse kinematics analysis is very important since it is the base of trajectory planning and realtime control. It is the best result to obtain analytical solutions of the inverse kinematic problem for it can well meet the requirements of real-time control. In the case of a serial manipulator with six degrees of freedom, it must meet one of the following two conditions to have analytical solutions of its inverse kinematic problem. One is that it has three adjacent parallel joints. The other is that it has three adjacent joints whose axes intersect at one point. HydM meets the first condition, so we can obtain analytical solutions of its inverse kinematics.

Generally, the position and posture of the end-effector are given with homogeneous transformation matrix shown as Eq. (2). And the vector $\left[p_{x}^{\prime}, p_{y}^{\prime}, p_{z}^{\prime}\right]^{\mathrm{T}}$ gives the position while the $3 \times 3$ matrix at the upper left corner of ${ }^{0} \mathbf{T}_{6}^{\prime}$ gives the posture of the end-effector in global frame $\left\{O_{0}\right\}$.

${ }^{0} \mathbf{T}_{6}^{\prime}=\left[\begin{array}{cccc}n_{x}^{\prime} & o_{x}^{\prime} & a_{x}^{\prime} & p_{x}^{\prime} \\ n_{y}^{\prime} & o_{y}^{\prime} & a_{y}^{\prime} & p_{y}^{\prime} \\ n_{z}^{\prime} & o_{z}^{\prime} & a_{z}^{\prime} & p_{z}^{\prime} \\ 0 & 0 & 0 & 1\end{array}\right]$

According to the homogeneous transformation rules, the given position and posture of the end-effector described in frame $\left\{O_{1}\right\}$ can be shown with Eq. (3). Simultaneously, the same position and posture of the end-effector can be shown with Eq. (4) due to the forward kinematics.

${ }^{1} \mathbf{T}_{6}^{\prime}={ }^{0} \mathbf{T}_{1}^{-1} \cdot{ }^{-0} \mathbf{T}_{6}^{\prime}=\left[\begin{array}{cccc}n_{x}^{\prime} c_{1}+n_{y}^{\prime} s_{1} & o_{x}^{\prime} c_{1}+o_{y}^{\prime} s_{1} & a_{x}^{\prime} c_{1}+a_{y}^{\prime} s_{1} & p_{x}^{\prime} c_{1}+p_{y}^{\prime} s_{1} \\ -n_{x}^{\prime} s_{1}+n_{y}^{\prime} c_{1} & -o_{x}^{\prime} s_{1}+o_{y}^{\prime} c_{1} & -a_{x}^{\prime} s_{1}+a_{y}^{\prime} c_{1} & -p_{x}^{\prime} s_{1}+p_{y}^{\prime} c_{1} \\ n_{z}^{\prime} & o_{z}^{\prime} & a_{z}^{\prime} & p_{z}^{\prime}-d_{1} \\ 0 & 0 & 0 & 1\end{array}\right]$ 


$$
\begin{aligned}
& { }^{1} \mathbf{T}_{6}={ }^{1} \mathbf{T}_{2}{ }^{2} \mathbf{T}_{3}{ }^{3} \mathbf{T}_{4}{ }_{4}^{4} \mathbf{T}_{5}{ }^{5} \mathbf{T}_{6} \\
& =\left[\begin{array}{cccc}
c_{234} c_{5} c_{6}+s_{234} s_{6} & -c_{234} c_{5} s_{6}+s_{234} c_{6} & -c_{234} s_{5} & a_{2} c_{2}+a_{3} c_{23}+a_{4} c_{234}-d_{5} s_{234}-d_{6} c_{234} s_{5} \\
s_{5} c_{6} & -s_{5} s_{6} & c_{5} & d_{6} c_{5} \\
s_{234} c_{5} c_{6}-c_{234} s_{6} & -s_{234} c_{5} s_{6}-c_{234} c_{6} & -s_{234} s_{5} & a_{2} s_{2}+a_{3} s_{23}+a_{4} s_{234}+d_{5} c_{234}-d_{6} s_{234} s_{5} \\
0 & 0 & 0 & 1
\end{array}\right]
\end{aligned}
$$

The Eqs. (3) and (4) are all describe the position and posture of the end-effector in frame $\left\{O_{1}\right\}$, so the corresponding elements of the two matrices should be equal to each other. The three elements $(1,4),(2,4)$ and $(3,4)$ in homogeneous matrix decide the position of end-effector, while as elements $(1,3),(2,3)$ and $(3,3)$ decide the posture of $z$ axis of the end-effector in a given frame. But the three elements $(1,3),(2,3)$ and $(3,3)$ are not independent, so an element in other columns should be selected to decide the posture of $x$ - or $y$-axis of the end-effector. The seven pairs of corresponding elements $(1,4),(2,4),(3,4),(1,3),(2,3),(3$, $3)$ and $(2,1)$ in matrices ${ }^{1} \mathbf{T}_{6}$ and ${ }^{1} \mathbf{T}_{6}^{\prime}$ are selected and listed as following:

$$
\begin{aligned}
& a_{2} c_{2}+a_{3} c_{23}+a_{4} c_{234}-d_{5} s_{234}-d_{6} c_{234} s_{5}=p_{x}^{\prime} c_{1}+p_{y}^{\prime} s_{1} \\
& d_{6} c_{5}=-p_{x}^{\prime} s_{1}+p_{y}^{\prime} c_{1} \\
& a_{2} s_{2}+a_{3} s_{23}+a_{4} s_{234}+d_{5} c_{234}-d_{6} s_{234} s_{5}=p_{z}^{\prime}-d_{1} \\
& -c_{234} s_{5}=a_{x}^{\prime} c_{1}+a_{y}^{\prime} s_{1} \\
& c_{5}=-a_{x}^{\prime} s_{1}+a_{y}^{\prime} c_{1} \\
& -S_{234} s_{5}=a_{z}^{\prime} \\
& S_{5} c_{6}=-n_{x}^{\prime} s_{1}+n_{y}^{\prime} c_{1}
\end{aligned}
$$

From Eqs. (6) and (9), we have

$$
\left(-a_{x}^{\prime} s_{1}+a_{y}^{\prime} c_{1}\right) d_{6}=-p_{x}^{\prime} s_{1}+p_{y}^{\prime} c_{1}
$$

then

$\theta_{1}=\arctan \frac{p_{y}^{\prime}-d_{6} a_{y}^{\prime}}{p_{x}^{\prime}-d_{6} a_{x}^{\prime}}$

From Eq. (9), we have

$\theta_{5}=-\arccos \left(-a_{x}^{\prime} s_{1}+a_{y}^{\prime} c_{1}\right)$

Since $\sin \theta_{5} \neq 0$, so Eqs. (8) and (10) can be re-written as

$$
\begin{aligned}
& c_{234}=-\frac{a_{x}^{\prime} c_{1}+a_{y}^{\prime} s_{1}}{s_{5}} \\
& S_{234}=-\frac{a_{z}^{\prime}}{s_{5}}
\end{aligned}
$$

Substitute Eqs. (15) and (16) into Eqs. (5) and (7), then

$$
a_{2} c_{2}+a_{3} c_{23}=p_{x}^{\prime} c_{1}+p_{y}^{\prime} s_{1}+a_{4} \frac{a_{x}^{\prime} c_{1}+a_{y}^{\prime} s_{1}}{s_{5}}-d_{5} \frac{a_{z}^{\prime}}{s_{5}}-d_{6}\left(a_{x}^{\prime} c_{1}+a_{y}^{\prime} s_{1}\right)
$$

$a_{2} s_{2}+a_{3} s_{23}=p_{z}^{\prime}-d_{1}+a_{4} \frac{a_{z}^{\prime}}{s_{5}}+d_{5} \frac{a_{x}^{\prime} c_{1}+a_{y}^{\prime} s_{1}}{s_{5}}-d_{6} a_{z}^{\prime}$

Define

$m=p_{x}^{\prime} c_{1}+p_{y}^{\prime} s_{1}+a_{4} \frac{a_{x}^{\prime} c_{1}+a_{y}^{\prime} s_{1}}{s_{5}}-d_{5} \frac{a_{z}^{\prime}}{s_{5}}-d_{6}\left(a_{x}^{\prime} c_{1}+a_{y}^{\prime} s_{1}\right)$

$n=p_{z}^{\prime}-d_{1}+a_{4} \frac{a_{z}^{\prime}}{s_{5}}+d_{5} \frac{a_{x}^{\prime} c_{1}+a_{y}^{\prime} s_{1}}{s_{5}}-d_{6} a_{z}^{\prime}$

Then, Eqs. (5) and (7) can be re-written as

$a_{2} c_{2}+a_{3} c_{23}=m$

$a_{2} s_{2}+a_{3} s_{23}=n$

We can get an equation only included $c_{3}$ by adding the two sides square of Eq. (19) to those of Eq. (20) respectively.

$$
2 a_{2} a_{3} c_{3}=m^{2}+n^{2}-a_{2}^{2}-a_{3}^{2}
$$

then

$\theta_{3}=-\arccos \frac{m^{2}+n^{2}-a_{2}^{2}-a_{3}^{2}}{2 a_{2} a_{3}}$

Eqs. (19) and (20) can be transformed to the following two equations due to the trigonometric function algorithm.

$$
\begin{aligned}
& \left(a_{2}+a_{3} c_{3}\right) c_{2}-a_{3} s_{3} s_{2}=m \\
& a_{3} s_{3} c_{2}+\left(a_{2}+a_{3} c_{3}\right) s_{2}=n
\end{aligned}
$$

From Eqs. (23) and (24), we can eliminate $s_{2}$ and get

$\left[\left(a_{2}+a_{3} c_{3}\right)^{2}+\left(a_{3} s_{3}\right)^{2}\right] c_{2}=m\left(a_{2}+a_{3} c_{3}\right)+n a_{3} s_{3}$

then

$\theta_{2}=\arccos \frac{m\left(a_{2}+a_{3} c_{3}\right)+n a_{3} s_{3}}{\left(a_{2}+a_{3} c_{3}\right)^{2}+\left(a_{3} s_{3}\right)^{2}}$

According to the homogeneous transformation method, the position and posture of the end-effector described in frame $\left\{\mathrm{O}_{3}\right\}$ can be derived as the following matrix:

$$
\begin{aligned}
& { }^{3} \mathbf{T}_{6}={ }^{3} \mathbf{T}_{4}{ }^{4} \mathbf{T}_{5}{ }^{5} \mathbf{T}_{6}= \\
& {\left[\begin{array}{cccc}
c_{4} c_{5} c_{6}+s_{4} s_{6} & -c_{4} c_{5} s_{6}+s_{4} c_{6} & -c_{4} s_{5} & a_{3}+a_{4} c_{4}-d_{5} s_{4}-d_{6} c_{4} s_{5} \\
s_{4} c_{5} c_{6}-c_{4} s_{6} & -s_{4} c_{5} s_{6}-c_{4} c_{6} & -s_{4} s_{5} & a_{4} s_{4}+d_{5} c_{4}-d_{6} s_{4} s_{5} \\
-s_{5} c_{6} & s_{5} s_{6} & -c_{5} & -d_{6} c_{5} \\
0 & 0 & 0 & 1
\end{array}\right]} \\
& {\left[\begin{array}{c}
{ }^{3} p_{6}^{\prime}={ }^{2} \mathbf{T}_{3}^{-1} \cdot{ }^{1} \mathbf{T}_{2}^{-1} \cdot{ }^{0} \mathbf{T}_{1}^{-1} \cdot{ }^{0} p_{6}^{\prime}= \\
a_{2} c_{3}+p_{x}^{\prime} c_{1} c_{23}+p_{y}^{\prime} s_{1} c_{23}+p_{z}^{\prime} s_{23}-d_{1} s_{23} \\
a_{2} s_{3}-p_{x}^{\prime} c_{1} s_{23}-p_{y}^{\prime} s_{1} s_{23}+p_{z}^{\prime} c_{23}-d_{1} c_{23} \\
p_{x}^{\prime} s_{1}-p_{y}^{\prime} c_{1} \\
1
\end{array}\right]}
\end{aligned}
$$


Eq. (27) shows the given position of the end-effector described in frame $\left\{O_{3}\right\}$. It should be equal to the fourth column of the position and posture matrix deduced from the forward kinematics shown as Eq. (28). Therefore, we can get two equations as below:

$a_{3}+\left(a_{4}-d_{6} s_{5}\right) c_{4}-d_{5} s_{4}=-a_{2} c_{3}+p_{x}^{\prime} c_{1} c_{23}+p_{y}^{\prime} s_{1} c_{23}+p_{z}^{\prime} s_{23}-d_{1} s_{23}$

$\left(a_{4}-d_{6} s_{5}\right) s_{4}+d_{5} c_{4}=a_{2} s_{3}-p_{x}^{\prime} c_{1} s_{23}-p_{y}^{\prime} s_{1} s_{23}+p_{z}^{\prime} c_{23}-d_{1} c_{23}$

Define

$u=-a_{2} c_{3}-a_{3}+p_{x}^{\prime} c_{1} c_{23}+p_{y}^{\prime} s_{1} c_{23}+p_{z}^{\prime} s_{23}-d_{1} s_{23}$

$v=a_{2} S_{3}-p_{x}^{\prime} c_{1} S_{23}-p_{y}^{\prime} s_{1} S_{23}+p_{z}^{\prime} c_{23}-d_{1} c_{23}$

Then, Eqs. (29) and (30) can be re-written as

$\left(a_{4}-d_{6} s_{5}\right) c_{4}-d_{5} s_{4}=u$

$\left(a_{4}-d_{6} s_{5}\right) s_{4}+d_{5} c_{4}=v$

From Eqs. (31) and (32), we can eliminate $c_{4}$ and get

$\left[\left(a_{4}-d_{6} s_{5}\right)^{2}+d_{5}^{2}\right] s_{4}=v\left(a_{4}-d_{6} s_{5}\right)-u d_{5}$

Then

$\theta_{4}=\arcsin \frac{v\left(a_{4}-d_{6} s_{5}\right)-u d_{5}}{\left(a_{4}-d_{6} s_{5}\right)^{2}+d_{5}^{2}}$

From Eq. (11), we can get $\theta_{6}$ as

$\theta_{6}= \pm \arccos \frac{-n_{x}^{\prime} s_{1}+n_{y}^{\prime} c_{1}}{s_{5}}$

According to the DH rules, the plus sign should be selected for the right side of Eq. (35) if $\cos \left(x_{6} \cdot z_{5}\right)$ is nonpositive, otherwise the minus sign should be selected. And $\cos \left(x_{6} \cdot z_{5}\right)$ can be derived from the following equation:

$$
\begin{aligned}
& {\left[\begin{array}{c}
\cos \left(x_{6} \cdot x_{5}\right) \\
\cos \left(x_{6} \cdot y_{5}\right) \\
\cos \left(x_{6} \cdot z_{5}\right)
\end{array}\right]={ }^{4} R_{5}^{T 3} R_{4}^{T 2} R_{3}^{T 1} R_{2}^{T 0} R_{1}^{T}\left[\begin{array}{c}
n_{x}^{\prime} \\
n_{y}^{\prime} \\
n_{z}^{\prime}
\end{array}\right]=} \\
& {\left[\begin{array}{c}
n_{x}^{\prime}\left(c_{1} c_{234} c_{5}-s_{1} s_{5}\right)+n_{y}^{\prime}\left(s_{1} c_{234} c_{5}+c_{1} s_{5}\right)+n_{z}^{\prime} s_{234} c_{5} \\
-n_{x}^{\prime}\left(c_{1} c_{234} s_{5}+s_{1} c_{5}\right)-n_{y}^{\prime}\left(s_{1} c_{234} s_{5}-c_{1} c_{5}\right)-n_{z}^{\prime} s_{234} s_{5} \\
-n_{x}^{\prime} c_{1} s_{234}-n_{y}^{\prime} s_{1} s_{234}+n_{z}^{\prime} c_{234}
\end{array}\right]}
\end{aligned}
$$

\subsection{The Given Position and Posture of the End-Effector}

The end-effector should approach to the objective in an optional posture and a series of planned posture in processing procedure. So, the position and posture of the end-effector are known and they are generally given with a homogeneous transformation matrix shown as Eq. (2). The position vector $\left[p_{x}^{\prime}, p_{y}^{\prime}, p_{z}^{\prime}\right]^{\mathrm{T}}$ can be given directly according to the position of the objective in global frame $\left\{O_{0}\right\}$. The posture of the end-effector can be given with $z-y-z$ Euler angles shown in Fig. (3) [11]. In fact, $\alpha$ and $\beta$ have obvious geometric meanings and are easy to measure. $\alpha$ is the included angle between axis $x_{0}$ and the projection line in $x_{0} y_{0}$ plane of axis $z_{6} . \beta$ is the included angle between axes $z_{0}$ and $z_{6}$.

From Fig. (3), it can be concluded that the frame $\left\{O_{6}\right\}$ is transformed from the original posture duplicated with the global frame $\left\{O_{0}\right\}$ through three rotation transformations. The first one makes $\left\{O_{6}\right\}$ to rotate $\alpha$ around axis $z_{6}$ to $\left\{O_{6}^{\prime}\right\}$. The second one makes $\left\{O_{6}^{\prime}\right\}$ to rotate $\beta$ around axis $y_{6}^{\prime}$ to $\left\{O_{6}^{\prime \prime}\right\}$. The last one makes $\left\{O_{6}^{\prime \prime}\right\}$ to rotate $\gamma$ around $z_{6}^{\prime \prime}$ to $\left\{O_{6}^{\prime \prime \prime}\right\}$. The posture of $\left\{O_{6}^{\prime \prime \prime}\right\}$ is the given posture of the endeffector which is shown as Eq. (37).

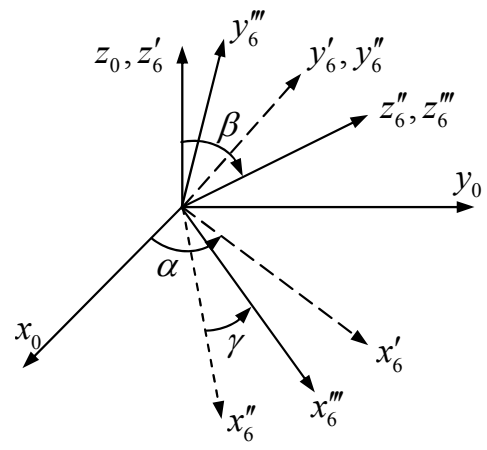

Fig. (3). $z-y-z$ Euler angles.

${ }^{0} R_{6}^{\prime}=\operatorname{Rot}(z, \alpha) \operatorname{Rot}(y, \beta) \operatorname{Rot}(z, \gamma)=$

$$
\left[\begin{array}{ccc}
c \alpha c \beta c \gamma-s \alpha s \gamma & -c \alpha c \beta s \gamma-s \alpha c \gamma & c \alpha s \beta \\
c \alpha s \beta c \gamma+c \alpha s \gamma & -s \alpha c \beta s \gamma+c \alpha c \gamma & s \alpha s \beta \\
-s \beta c \gamma & s \beta s \gamma & c \beta
\end{array}\right]
$$

\subsection{The Correctness Verification of the Inverse Solution}

The block diagram in Simulink for verifying the correctness of the inverse solution is shown in Fig. (4). Firstly, a certain number of sets of joint variables are produced randomly in their limits. Then the corresponding

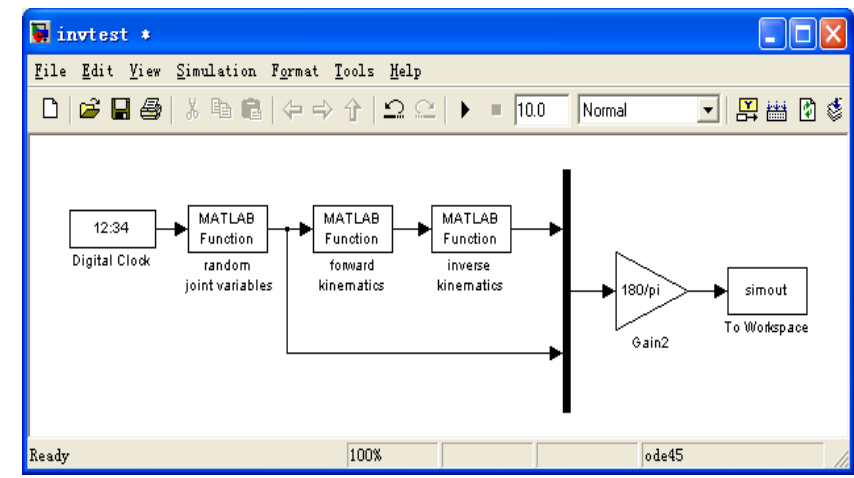

Fig. (4). Block diagram for inverse solution verification. 
positions and postures of the end-effector are calculated. The next procedure is to obtain the corresponding inverse solutions through the inverse kinematic equations. The last procedure is to compare the inverse solutions and the random joint variables. If they are equal to each other within a certain precision, it can be concluded that the inverse solutions are correct.

\section{CONCLUSION}

This paper gives a mechanism design of a six DOF hydraulically actuated manipulator which has some obvious advantages compared to traditional electric powered manipulators. Those are with high power-mass ratio, electric insulation, suitable for severe tasks, etc. So it can be used in underwater, electric power line maintenance, forest, construction, nuclear and other industries. Then the analytical solutions of its inverse kinematics are solved by given the position and posture of the end-effector simultaneously. The posture of the end-effector is given through three $z-y-z$ Euler angles for they have obvious geometry meanings and are easy to be measured. The solving method of inverse kinematics and the posture given method of the end-effector can provide important references to those with similar configuration to it.

\section{CONFLICT OF INTEREST}

The authors confirm that this article content has no conflict of interest.

\section{ACKNOWLEDGEMENTS}

This work is supported by the High Technology Research and Development Program of China under grant no. 2012AA041506.

\section{REFERENCES}

[1] K. Ahn, and S. Yokota, "Application of discrete event control to the insertion task of electric line using 6-link electro-hydraulic manipulators with dual arm", JSME International Journal, Series C: Mechanical Systems, Machine Elements and Manufacturing, vol. 46, no. 1, pp. 263-269, 2003.

[2] L. Q. Cai, "Hydraulic manipulator system for live electric line operation", Construction Machinery for Hydraulic Engineering \& Power Stations, vol. 16, no. 2, pp. 42-48, 1994. (In Chinese)

[3] M. W. Dunnigan, D. M. Lane, A. C. Clegg, and I. Edwards, "Hybrid position/force control of a hydraulic underwater manipulator", IEE Proceedings: Control Theory and Applications, vol. 143, no. 2, pp. 145-151, 1996.

[4] P. M. La Hera, U. Mettin, S. Westerberg, and A. S. Shiriaev, "Modeling and control of hydraulic rotary actuators used in forestry cranes", In: Proceedings of the IEEE International Conference on Robotics and Automation, 2009, pp. 1315-1320.

[5] R. F. Abo-Shanab, and N. Sepehri, "On dynamic stability of manipulators mounted on mobile platforms", Robotica, vol. 19, no. 4, pp. 439-449, 2001.

[6] C. J. Taylor, and D. Robertson, "State-dependent control of a hydraulically actuated nuclear decommissioning robot", Control Engineering Practice, vol. 21, no. 12, pp. 1716-1725, 2013.

[7] M. J. Bakari, K. M. Zied, and D. W. Seward, "Development of a multi-arm mobile robot for nuclear decommissioning tasks", International Journal of Advanced Robotic Systems, vol. 4, no. 4, pp. 387-406, 2007.

[8] X. Y. Wang, R. Liu, and G. Z. Jia, "On hydraulic manipulator control with generalization pulse code modulation", Chinese Journal of Mechanical Engineering, vol. 38 (supp.), pp. 203-206, 2002. (In Chinese)

[9] Y. L. Zhao, S. Y. Lu, X. C. Lv, J. Li, and Z. L. Wang, "Design of force feedback hydraulic arm for live working robot", Chinese Hydraulics \& Pneumatics, no. 8, pp. 31-34, 2013. (In Chinese)

[10] Y. L. Zhao, Q. Zhang, H. Qi, J. Li, and Y. C. Li, "Design of non-force feedback hydraulic arm for live working", Machinery Design \& Manufacture, no. 9, pp. 117-119, 2012. (In Chinese)

[11] J. J. Craig, Introduction to Robotics: Mechanics and Control (3rd Edition), Prentice Hall Press, 2004. 